\title{
EFFECTS OF SUSCEPTOR GEOMETRY ON GAN GROWTH ON SI(111) WITH A NEW MOCVD REACTOR
}

\author{
Yungeng Gao,* Daniel A. Gulino,* and Ryan Higgins** \\ * Department of Chemical Engineering and Condensed Matter and Surface Science Program, \\ Ohio University, Athens, OH 45701 \\ ** Condensed Matter and Surface Science Program, Ohio University, Athens, OH 45701
}

Cite this article as: MRS Internet J. Nitride Semicon. Res. 4S1, G3.53(1999)

\begin{abstract}
High quality GaN films on AlN buffer layers were grown on $\mathrm{Si}(111)$ with a new, commercial, two-injector vertical rotating disk MOCVD reactor (CVD, Inc.). It was found that the geometry of the susceptor greatly affected the structural quality of the epilayers on $\mathrm{Si}$. For the original susceptor geometry, though single crystal $\mathrm{GaN}$ films could be obtained, the films were dark gray in appearance with a rough morphology, and the best x-ray rocking curve FWHM was $2.33^{\circ}$. After modifying the susceptor geometry, transparent, mirror-like single crystal GaN films were obtained with the best x-ray rocking curve FWHM being $0.24^{\circ}$. Photoluminescence (PL) and infrared reflectance (IR) spectra of the grown films were compared. The film growth rate was found to increase with decrease of the growth pressure. A 2-D simulation of the flow, heat transfer, and chemical species transport in the reactor showed a more symmetric flow, larger velocity gradient, and lower upward velocity with the modified susceptor, which may be the main reason for the improvement of the structural quality of the films.
\end{abstract}

\section{INTRODUCTION}

GaN has attracted much research interest due to its potential application in optical, high temperature, and high power devices. Although high quality $\mathrm{GaN}$ films have been achieved by MOCVD growth, the mechanism is still not fully understood. Factors found to affect MOCVD growth of GaN include substrate properties, ${ }^{[1]}$ any buffer layer and its growth conditions, ${ }^{[2]}$ epilayer growth temperature and pressure, ${ }^{[3]} \mathrm{V} / \mathrm{III}$ molar flow ratio, ${ }^{[4]}$ inlet flow rate and reactor configuration, ${ }^{[5]}$ among others, but a careful control of these factors does not guarantee a high quality film. The growth of GaN on silicon is very difficult due to their large difference in lattice constant, crystal structure, and thermal expansion coefficient, ${ }^{[6]}$ yet silicon is a very attractive material since it offers the possibility of incorporating GaN devices in silicon devices. In this work, a new, commercial, two-injector, vertical rotating disk MOCVD reactor (CVD Equipment Corporation) was used to grow $\mathrm{GaN}$ on $\mathrm{Si}(111)$. After experiencing difficulties with film growth using the standard geometry, we modified it and found that the susceptor geometry greatly affected the structural quality of the films.

\section{EXPERIMENT}

A new, commercial MOCVD system (CVD, Inc.) was used for GaN growth. It consists of a gas delivery system, pumping system, and reaction chamber. The reaction chamber is schematically illustrated in Figure 1a. It is a two-injector, vertical, cold-wall reactor with a rotating molybdenum susceptor inductively heated by RF. The susceptor is capable of processing one wafer with a diameter of 1 inch per run. A thermocouple is placed inside a quartz shaft underneath the susceptor 


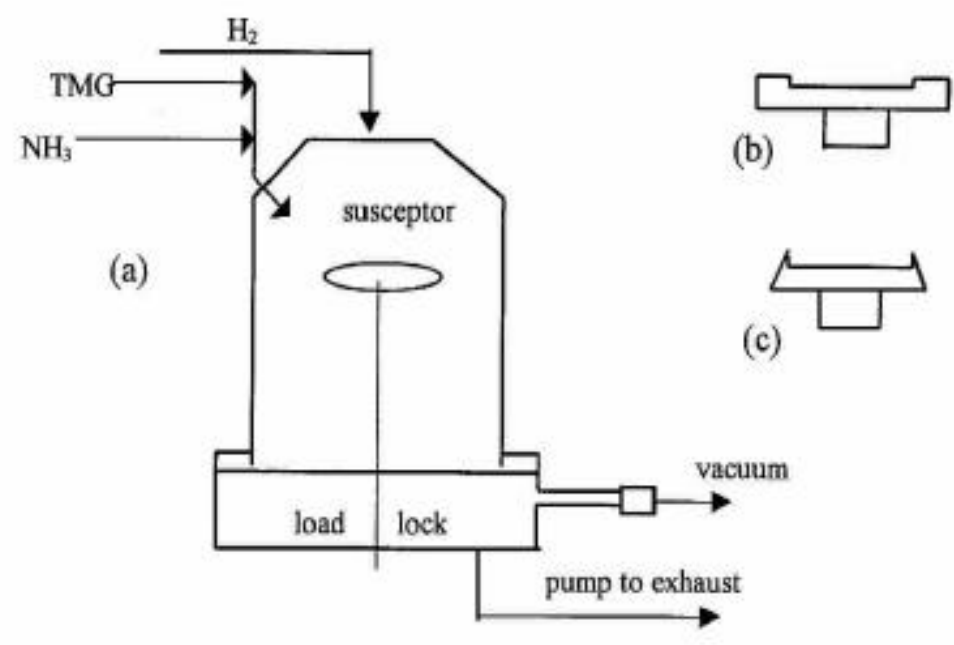

Figure 1. (a) schematic of the MOCVD reaction chamber. (b) original susceptor shape. (c) modified susceptor shape. to monitor the growth temperature. A closed loop vacuum pumping system enables the reactor to be operated over a pressure range of 10 to 760 torr with good stability. A high vacuum loadlock is interfaced to the reactor to isolate it from the ambient environment during wafer loading and unloading operations. Figures 1(b) and (c) show the original and modified susceptor, respectively. The original, manufacturer-supplied susceptor has a diameter of

$37.5 \mathrm{~mm}$, including a circular central part (which has a diameter of $25.5 \mathrm{~mm}$ ) and an outer edge of $6 \mathrm{~mm}$ width. The central part of the modified susceptor was unchanged from the original, but the outer edge was cut and tapered. The diameter at the base of the modified susceptor was $31.5 \mathrm{~mm}$.

The source gases were ammonia $\left(\mathrm{NH}_{3}\right)$, trimethylgallium (TMGa), and trimethylaluminum (TMA). Hydrogen was used as a carrier gas. Silicon (111) was used as the substrate, and it was cleaned in acetone, $2 \% \mathrm{HF}$, and UHP deionized water, then dried with $\mathrm{N}_{2}$. Prior to growth, it was heated at $1050^{\circ} \mathrm{C}$ under a hydrogen flow of 2 slpm for $5 \mathrm{~min}$. A total inlet gas flow rate of $1.0 \mathrm{slm}$ was used. The inlet flow distribution was 0.4 slpm $\mathrm{H}_{2}, 0.3 \mathrm{slpm} \mathrm{NH}_{3}$, and $0.3 \mathrm{slpm} \mathrm{H}_{2}$ to push the TMA or TMG. The carrier gas flow rate through the TMA bubbler $\left(28^{\circ} \mathrm{C}\right)$ or TMG bubbler $\left(-10^{\circ} \mathrm{C}\right.$ ) was $1 \mathrm{sccm}$. The calculated V/III molar flow ratio was 4390. An AlN buffer layer with a thickness of around $100 \mathrm{~nm}$ was grown at $1050^{\circ} \mathrm{C}$. ${ }^{[7]}$ The $\mathrm{GaN}$ epilayer was grown at $1000^{\circ} \mathrm{C}$ for 45 minutes. Four samples were grown under the above conditions, but with differing susceptor geometry, growth pressure, or susceptor rotation rate as shown in Table I. Sample 1 was the best of the samples deposited using the original susceptor.

Table I. Sample growth conditions.

\begin{tabular}{|r|c|c|c|c|}
\hline & sample 1 & sample 2 & sample 3 & sample 4 \\
\hline \hline susceptor shape & original & modified & modified & modified \\
\hline pressure (torr) & 76 & 10 & 10 & 76 \\
\hline spin rate (rpm) & 60 & 150 & 60 & 150 \\
\hline
\end{tabular}

X-ray diffraction (XRD), Photoluminescence (PL), and Infrared Reflectance (IR) were used to characterize the deposited films. XRD was performed with a Rigaku X-ray diffractometer with a wide angle automated goniometer and computer-based data acquisition and analysis system. IR data was collected on a Perkin-Elmer Spectrum 2000 spectrometer using unpolarized light with a reflectance angle of $0^{\circ}$ from the normal. A gold mirror was used as the reference. PL emission at 9.5K was measured. A He-Cd laser beam $(325 \mathrm{~nm}, 9 \mathrm{mw})$ was used as the excitation source. The 


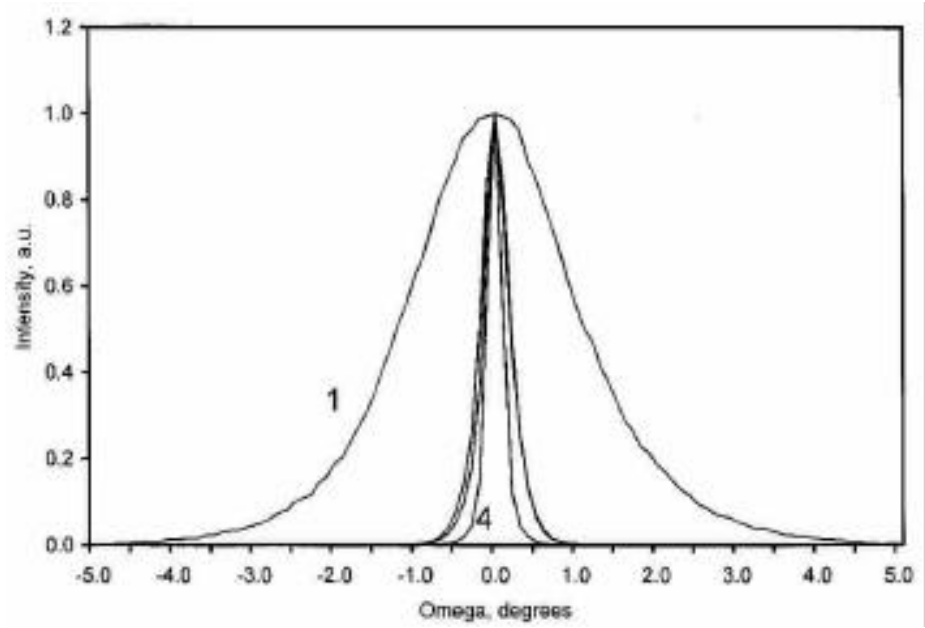

optical signal was detected by Princeton Instruments back-illuminated CCD camera model TEA-CCD-512 TK with a UV/AR coating and controlled by a computer. Commercial CFD software (FLUENT) was used to characterize the fluid dynamics in the reactor.

Figure 2. X-ray rocking curves on the GaN (0002) reflection. The curves for samples 1 and 4 are labeled.

\section{RESULTS}

The appearance of sample 1 was rough and dark gray, while samples 2 through 4 appeared transparent and mirror-like.

\subsection{XRD Results}

Theta-2 theta scans of the samples showed that, in addition to Si reflections, only four other reflections were detectable, i.e. GaN (0002), AlN (0002), GaN (0004), and AlN (0004). This showed that the epilayers were single crystal and in the wurtzite phase. Figure 2 is the $\mathrm{X}$-ray rocking curve on the GaN (0002) reflection. The FWHM of samples 1 to 4 were $2.33^{\circ}, 0.36^{\circ}, 0.40^{\circ}$, and $0.24^{\circ}$, respectively (shown in order 1, 3, 2, 4 in the figure with curves for samples 1 and 4 labeled), showing that the structural quality of the GaN films was much improved using the modified susceptor.

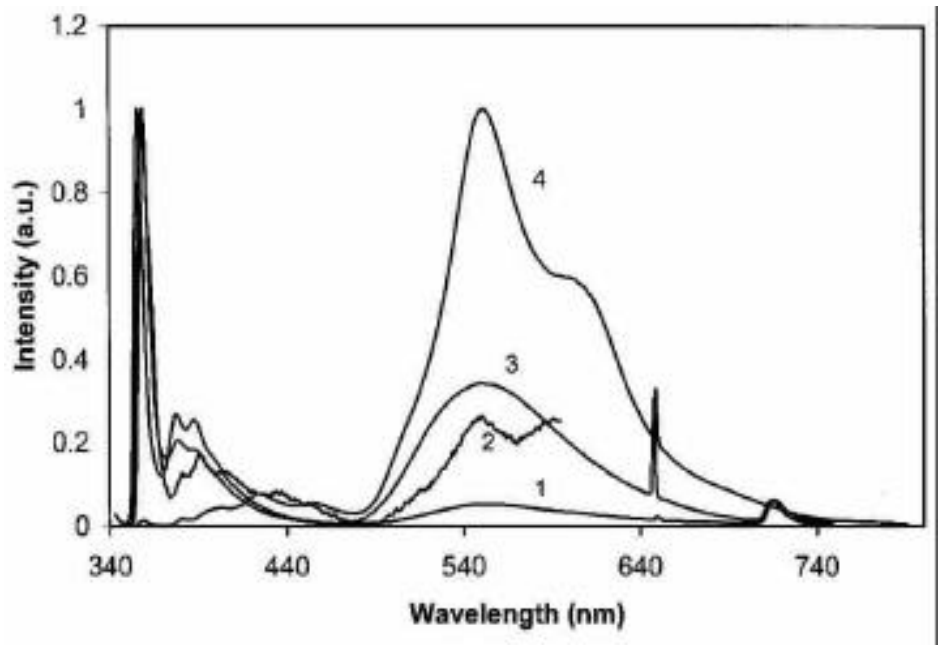

Figure 3. Photoluminescence spectra of the samples.

\subsection{PL Results}

Figure 3 shows the photoluminescence (PL) spectra of the samples. A very strong near band edge emission was observed at $358.5 \mathrm{~nm}$ on samples 1 and 2 and at $356.5 \mathrm{~nm}$ on sample 3. The FWHM of these peaks were 61,62 , and $59 \mathrm{meV}$, respectively. Two additional peaks at $378 \mathrm{~nm}$ and $388 \mathrm{~nm}$ were also observed on these samples. Sample 4 showed a very weak near band edge emission but a very strong yellow luminescence, which is surprising considering that the X-ray 
rocking curve of this sample was the narrowest.

\subsection{IR Results}

From the number of interference fringes that occurred between two frequencies of the IR transmittance spectra as seen in Figure 4, we calculated the thickness of the films, ${ }^{[8]}$ which were $1.12,1.27,1.32$, and $0.95 \mu \mathrm{m}$, respectively, for samples 1 to 4 . It was interesting to note the thickness (growth rate) differences among samples 2 to 4 . The growth rates under different susceptor spin rates (60 rpm vs. $150 \mathrm{rpm}$ )

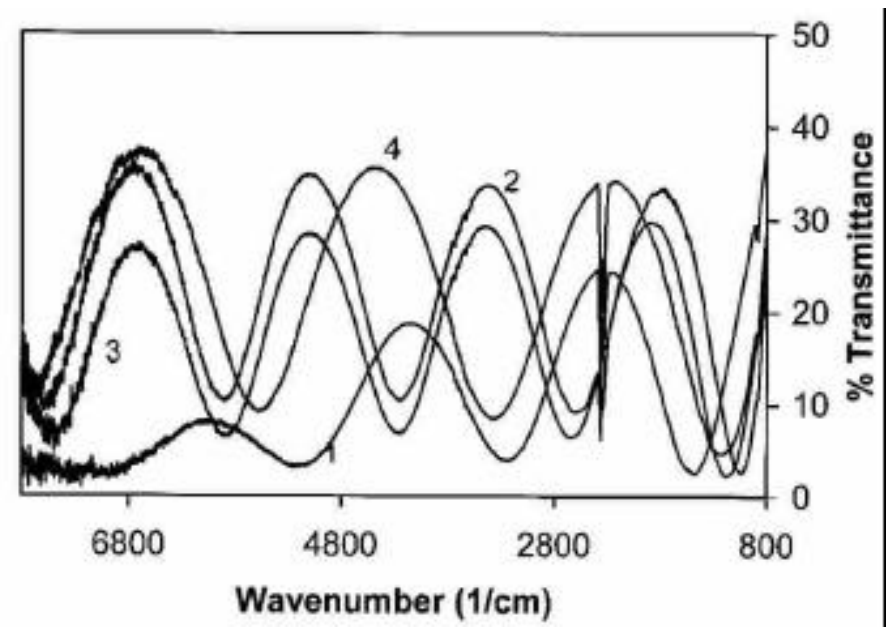

Figure 4. IR transmittance spectra. were almost the same, while there was a significant difference among the growth rates under different growth pressures, and the growth rate decreased with increasing pressure. This result was also confirmed by the thickness of sample 1, which was thinner than samples 2 or 3 . From Figure 4 we

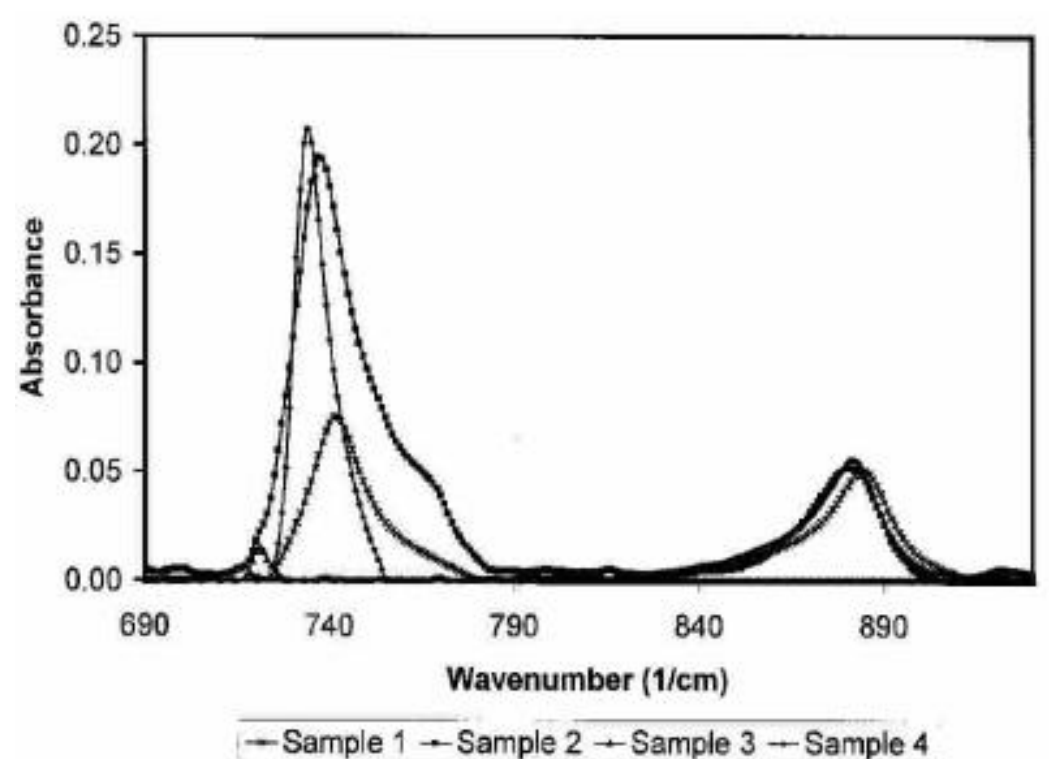

Figure 5. IR absorbance spectra. can also see that the interference fringes of sample 1 attenuate quickly at higher frequencies, which indicates the inferior quality of the film. Figure 5 shows the IR absorbance spectra of the films. A strong, sharp LO phonon mode in wurtzite $\mathrm{GaN}$ is observed at 735 $\mathrm{cm}^{-1}$ on sample 4, which further confirms both the high quality of the film and the $\mathrm{x}$-ray rocking curve results. The LO phonon mode was also observed on sample 2 at $738 \mathrm{~cm}^{-1}$. The phonon mode of sample 1 is shifted to a higher frequency of $743 \mathrm{~cm}^{-1}$. The LO phonon mode of AlN was also observed at around $882 \mathrm{~cm}^{-1}$. No GaN or AlN LO phonon mode was observed on sample 3; the reason for this is not clear.

\subsection{Reactor Simulation Results}

The fluid dynamics in the reactor with the two different susceptors was simulated using a 2-D model. The model consisted of a numerical solution of the Navier-Stokes equations coupled with heat transfer and mass transport of chemical species using FLUENT software. The effects of variable gas properties and buoyancy was included, but did not include the rotation of susceptor and chemical reactions. A detailed 3-D simulation with susceptor rotation is being conducted and the results will be reported later. Figures 6 and 7 show the vertical velocity contour in the reactor with the only difference being susceptor geometry. From the figures, the following differences are noted: (1) the 


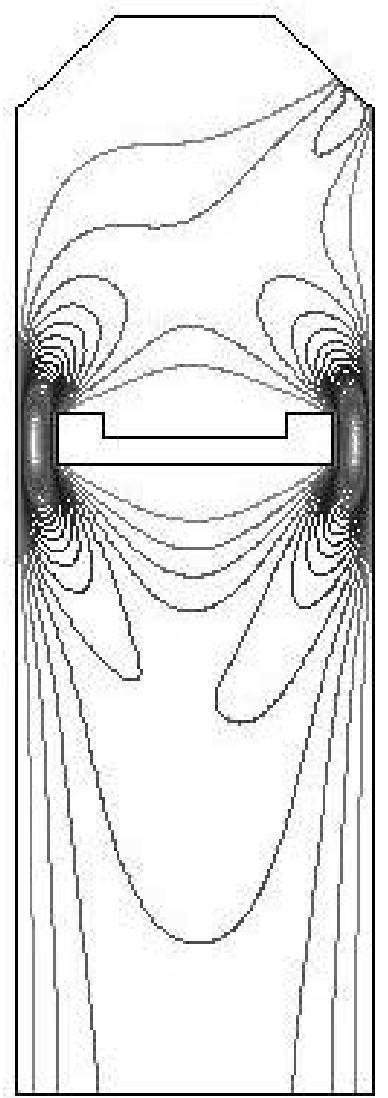

Figure 6. Vertical velocity contours for the original susceptor model.

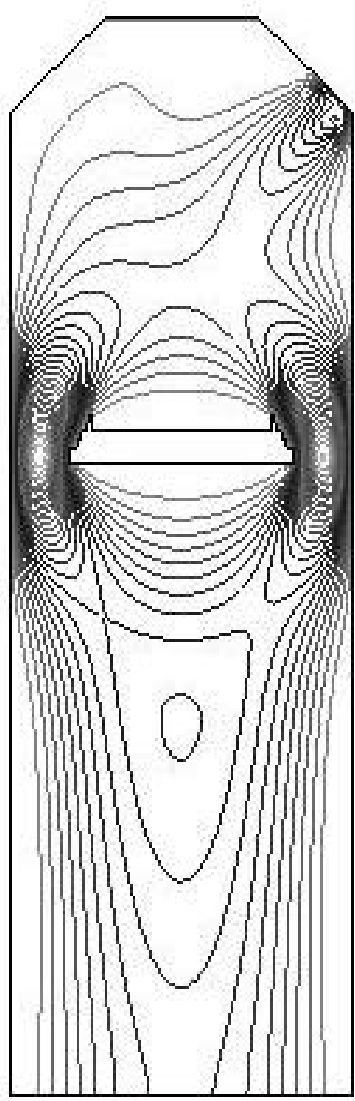

Figure 7. Vertical velocity contours for the modified susceptor model.

velocity distribution above the modified susceptor is more symmetric, and (2) the velocity gradient is larger above the modified susceptor. The model also indicates that the upward velocity above the original susceptor is more than twice that of the modified susceptor. (This is not discernable from the Figures.) The more symmetric flow, larger velocity gradient, and lower upward velocity may be the primary reasons for the improvement of the structural quality of the films.

\section{CONCLUSIONS}

The effect of susceptor geometry on the growth of GaN on silicon substrate using a new MOCVD system was studied. A modified susceptor was found to change the fluid dynamics in the reactor, resulting in a gas flow that is more symmetric, has a larger gradient, and has a weaker recirculation flow over the susceptor. The structural quality of films grown on the modified susceptor was much improved regardless of the susceptor rotation rate and growth pressure. Combining the PL and IR results, high structural and optical quality films could be obtained at 10 torr and $150 \mathrm{rpm}$ with the modified susceptor.

\section{ACKNOWLEDGMENTS}

Partial support for this work under grant BMDO-DURISP N00014-96-0782 is gratefully acknowledged. The authors wish to thank T. Lin, W. Jadwisienczak, H. Richardson, and B. Mehta at Ohio University for their help with both film and flow characterization.

\section{REFERENCES}


1. H. Morkoc, S. Strite, G. B. Gao, M. E. Lin, B. Sverdlov, and M. Burns, J. Appl. Phys. 76, 1363 (1994).

2. J. N. Kuznia, M. A. Khan, D. T. Olson, R. Kaplan, and J. Freitas, J. Appl. Phys. 73, 4700 (1993)

3. T. Sasaki, and T. Matsuoka, J. Appl. Phys. 77, 193 (1995).

4. M. Tchounkeu, O. Briot, B. Gil, J. P. Alexis, and R. L. Aulombard, J. Appl. Phys. 80, 352 (1996).

5. S. Nakamura, Y. Harada, and M. Seno, Appl. Phys. Lett.58, 2021 (1991)

6. G. A. Martin, B. N. Sverdlov, A. Botchkarev, H. Morkoc, D. J. Smith, S.-C. Y. Tsen, W. H. Thompson, M. H. Nayfeh, Mat. Res. Soc. Symp. Proc. 67, vol. 395 (1996).

7. A. Watanabe, T. Takcuchi, K. Hirosawa, H. Amano, K. Hiramatsu, and I. Akasaki, J. Crystal Growth 128, 391 (1993).

8. N. J. Harrick, Appl. Opt. 10, 2344 (1971). 